\title{
ON THE NUMERICAL CALCULATION OF THE DISTRIBUTION FUNCTIONS DEFINING SOME COMPOUND POISSON PROCESSES *)
}

\author{
CARL Philipson \\ Stockholm, Sweden
}

I. The compound Poisson process in the wide sense is defined as a process for which the probability distribution of the number $i$ of changes in the random function attached to the process, while the parameter passes from o to a fixed value $\tau$ of the parameter measured on a suitable scale, is given by the Laplace-Stieltjes integral

$$
\int_{0}^{\infty} e^{-v \tau}[(v \tau) i / i !] d_{v} U(v, \tau),
$$

where $U(v, \tau)$ for a fixed value of $\tau$ defines the distribution of $v . U(v, \tau)$ is called the risk distribution and is either $\tau$-independent or, dependent on $\tau$.

2. The compound Poisson process in the narrow sense is defined as a process for which the probability distribution of the number of changes can be written in the form of (I) with a $\tau$-independent risk distribution.

In their general form these processes have been analyzed by Ove Lundberg (I940) ${ }^{1}$ ). For such processes the following relation holds for the probability of $i$ changes in the interval o to $\tau, \bar{P}_{i}(\tau)$ say

$$
i ! \bar{P}_{i}(\tau)=(-\tau)^{i} \bar{P}_{0}^{(i)}(\tau)
$$

this relation does not hold for processes with $\tau$-dependent risk distribution. Hofmann (I955) has introduced a sub-set of the processes concerned in this section for which the probability for non-occurrence of a change in the interval $o$ to $\tau$ is defined as a solution of the differential equation

$$
y^{\prime} \bar{f}=y \bar{q}(\mathrm{I}+\tau / \bar{f})^{-x},
$$

*) Presented to the Colloquium I962 in Juan-les-Pins

1) Literature references are given at the end of the paper. 
$\bar{q}, \bar{f}>0$ and $x \geqslant 0$; the solutions may be written in the form ${ }^{(x)} \eta_{\tau}-\bar{q}$, where $\eta$ is independent of $\bar{q}$ and of two alternative forms one for $x=I$ and one for other values of $x$. The probabilities for $i$ changes in the interval $o$ to $\tau$ in the processes defined by the solutions of Hofmann's equation are derived by Leibniz's formula, and are designated by $\frac{(\kappa)}{q} \bar{\pi}_{i}(\tau)$ and, in this paper, called Hofmann probabilities.

For $x=0$ the Hofmann probabilities define a Poisson process, with application to the risk theory of insurance treated i.a. by Filip Lundberg, Cramér, Esscher, Segerdahl (for references see Philipson (I96I) (b)). For $x=\mathrm{I}$ the Hofmann probabilities define a Polya process, where the risk distribution is defined by a Pearson type III density with two parameters, which by normalization can be reduced to such a density with one parameter. Such processes have been treated by Ove Lundberg as applied to sickness and accident statistics, by Hofmann with the same scope of application, by Ammeter to reinsurance policy and other problems of general insurance, and by Campagne (I962) to conflagration between fire insurance risks. Delaporte has applied a modified form of the Polya distribution, where the risk distribution has three parameters, which cannot be reduced to such a distribution of I or 2 such parameters, to motor accident statistics [cf. Philipson (1960) (a) and for references (Ig6I) (b)]. The probability distribution of a Polya process with a risk distribution containing two parameters $\bar{q}, \bar{f}$ can be written

$$
{ }_{\bar{q}}^{(1)} \pi_{\imath}(\tau)=\left(\begin{array}{c}
-\bar{q} \\
i
\end{array}\right) \frac{(-\tau / \bar{f})^{2}}{(\mathbf{I}+\tau / \bar{f})^{i+\vec{q}}}
$$

$i=0, \mathrm{r}, 2 \ldots$ with mean $\frac{\tau \bar{q}}{\bar{f}}$.

The Hofmann probabilities are particular cases of a class of probabilities for non-occurrence of a change of the form

$$
\prod_{j=1}^{l} \eta_{j \tau}^{-q_{j}}
$$

where the $\eta_{j \tau}$ 's for all values of $j$ are independent of $q_{j}$ for $j=\mathbf{I}, 2$ 
and, in the general case, not necessarily in the form for $\eta$ in the Hofmann probabilities for non-occurrence. It shall be remarked that the $m^{\text {th }}$ power of a function of this class belongs to the class with the exponents equal to $-m q_{j}$. If all the $\eta_{j \tau}$ 's are in either of the alternative forms for Hofmann probabilities of non-occurrence the product may be said to define a generalized Hofmann probability, which may be designated $\left(x_{1}, x_{2}, \ldots x_{l}\right){ }_{q} \pi_{i}(\tau)={ }_{q} \pi_{i}(\tau)$ say.

3. The change distribution, $V(x, \tau)$, shall be written for the conditioned distribution function of the size of one change in a random function attached to a process, relative to the hypothesis that one such change has occurred in parameter interval $(\tau, \tau+d \tau)$ for a fixed value of $\tau$. (In the application to Life Insurance and to General Insurance this distribution function has been called the risk sum distribution and the claim distribution respectively. It seems to the author that these terms conceal that the theory has a much wider scope of application.) In the particular case, where $V(x, \tau)$ is independent of $\tau$, we shall write $V(x)$ for the change distribution.

Esscher (1932), Cramér (1955) and others have proved that the distribution functions defining a Poisson process with the change distribution $V(x, \tau)$ can be written

$$
\sum_{i=0}^{\infty}{ }_{1}^{00} \tilde{\pi}_{i}(t) W^{i *}(x, t),
$$

where $t$ is the parameter measured on the operational scale, $W(x, t)$ a transform of $V(x, \tau)$ and the asterisk power $i^{*}$ of any function for $i>0$ denotes the $i^{\text {th }}$ convolution of the function with itself and for $i=0$ is equal to unity. This formula has earlier been extended by the present author (Philipson, (I96I) (c)) to a formula of a similar form for the distribution function of a random function attached to a process defined by generalized Hofmann probabilities for non-occurrence of at most three factors, the restriction of the number of factors is, however, not likely to be necessary. ${ }^{1}$ )

1) In a report to the Congress in London, to be published it its Transactions, this formula has been extended for processes defined by (2) with the same transform $W(x, t)$ as for a Poisson process. 
4. A stationary or non-stationary compound Poisson process is defined as a process for which the probability distribution of the number of changes is defined by $(\mathrm{r})$ with $U(v, \tau)$ being in the form of the distribution functions defining (another) random process, called the primary process. Matérn (I960) has applied the stationary compound Poisson process, i.e. a process, where $U(v, \tau)$ is stationary in the weak sense, to sampling problems in forestry statistics, Thyrion has introduced a very general class of processes which contains both stationary and non-stationary compound Poisson processes. In this paper only such processes, generated by primary processes with $\tau$-independent change distributions, $U(v)$ say, shall be dealt with. Under this assumption $U(v, \tau)=\sum_{m=0}^{\infty} P_{m}(\tau) U^{m *}(v)$. In this case the probability distribution of the number $i$ of changes in the random function attached to a process defined by (I) can, as was previously found by the present author, be written in the form

$\sum_{m=0}^{\infty} P_{m}(\tau) \int_{0}^{\infty} e^{-v \tau}[(v \tau) i / i !] d U m *(v)=\sum_{m=0}^{\infty} P_{m}(\tau) \bar{P}_{i}(\tau, m)$, say.

The process, defined by (4), is said to be of a type defined by $\bar{P}_{i}(\tau, \mathrm{I})$. In the particular cases, where $\bar{P}_{i}(\tau, \mathrm{r})$ is defined by the Hofmann probabilities $\frac{(x)}{\bar{\tau}_{i}}(\tau)$ for $x=0, \mathrm{I}$, the process defined by (4) is of type Poisson for $x=0$ and of type Polya for $x=\mathrm{I}$. In these cases, using a Poisson distribution with one parameter and a Polya distribution with two parameters in the risk distribution, which is essential for the derivation, the functions $\vec{P}_{i}(\tau, m)$ take the following forms.

$\bar{P}_{i}(\tau, m)=\left\{\begin{array}{c}{\left[(m \bar{q} \tau)^{i} / i !\right] e^{-m \bar{q}} \quad \text { for } x=0 \text {, i.e. for type Poisson }} \\ \left(\begin{array}{c}-m \bar{q} \\ i\end{array}\right) \frac{(-\tau / \bar{f})^{i}}{(\mathrm{I}+\tau / \bar{f})^{i+m \bar{q}}} \text { for } x=\text { I, i.e. for type Polya }\end{array}\right.$

$A$ random function attached to processes defined by (4) and (5) and with $\tau$-independent change distribution $V(x)$ shall be designated by $Y^{*}(\tau)$.

5. A random function attached to a process, where the probability distribution of the number $m$ of changes is defined by the generalized 
Hofmann probabilities ${ }_{q} \pi_{m}(\tau)$ and where the change distribution depends on $\tau, V(x, \tau)$ say, and is such that its transform $W(x, \tau)$ as defined in section 3 , can be written in the form

$$
W(x, \tau)=\sum_{i=0}^{\infty} \frac{(x) \bar{l}}{\bar{q} \pi_{i}}(\tau) V^{i *}(x), \quad x=0, \mathrm{I}
$$

where $\frac{\left(x_{j}\right)}{q} \bar{\pi}_{i}(\tau)$ for $x=0, I$ has one respectively two parameters in the risk distribution and $V(x)$ is equal to that of $Y^{*}(\tau)$, shall be designated by $X^{*}(\tau)$.

$A$ random function attached to a compound Poisson process with a $\tau$-independent risk distribution, or such a process in the narrow sense, the probability distribution of the number $i$ of changes being defined by (I) with $U(v, \tau)=U(v)$ independently of $\tau$ and the change distribution by $V(x)$ independently of $\tau$, shall be designated by $\bar{X}^{*}(\tau)$.

6. Writing $q \tau$ for the means of both $P_{m}(\tau)$ and ${ }_{q} \pi_{m}(\tau), c$ for the mean of $V(x)$, the means of $Y^{*}(\tau)$ and $X^{*}(\tau)$ are both equal to $c q \bar{q} \tau^{2} / \bar{f}^{x}$ for $x=0$, I. If $\bar{q} \tau$ is the mean of $\bar{P}_{i}(\tau)$ defined by (r) with $U(v, \tau)=U(v)$ independently of $\tau$, the mean of $\bar{X}^{*}(\tau)$ is equal to $c \bar{q} \tau$. The normalization of the distribution functions of $Y^{*}(\tau)$, $X^{*}(\tau)$ and $\bar{X}^{*}(\tau)$ in order to render the means of the normalized functions equal to $t$ involves the substitution of $\left[\bar{f}^{x} t / c q \bar{q}\right]^{1 / 2}$ for $\tau$ in the two first cases and of $t / c \vec{q}$ for $\tau$ in the third case. After this transformation the random functions will be designated by $Y(t)$, $X(t)$ and $\bar{X}(t)$ respectively and their distribution functions for a fixed value of $t$ by ${ }_{Y}^{(x)} F(x, t),{ }_{X}^{(x)} F(x, t)$ and $\bar{X} F(x, t)$ respectively. Then, the following relation holds, where

$$
\begin{aligned}
& r_{0}=(c q \bar{q})^{\mathbf{1 / 2}} ; r_{1}=r_{0} / \bar{f}^{1 / 2} .
\end{aligned}
$$

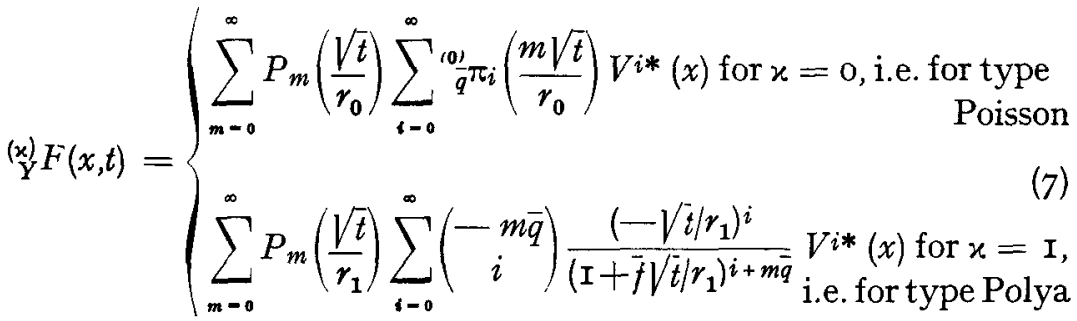

${ }_{X} F(x, t)$ has the form of $(7)$ with the substitution of ${ }_{q} \pi_{m}$ for $P_{m}$ 


$$
\bar{X} F(x, t)=\sum_{m=0}^{\infty} \bar{P}_{\imath}(t / c \bar{q}) V^{i *}(x)
$$

7. Almer (1957) has proved a theorem implying that every (statistical or theoretical) change distribution may-within a finite interval of its argument-be approximated by upper and lower approximations in the form of weighted sums of exponential distribution functions such that the area between the curves representing the upper and the lower approximation can be made as small as we want. He has graduated data from extensive motor accident statistics later extended to other branches of general insurance (Almer, I962) by such sums containing only 3 to 4 terms. These graduations showed a very good agreement with the data. His deductions (1957) lead to an expression for the distribution functions defining a Poisson process, with the change distribution defined by a weighted sum of exponential terms, in the form of a convolution of a limited number of Bessel functions.

8. Esscher (I96I-I962) has suggested that the change distribution shall be defined as a weighted sum of at least two exponential distribution functions and the distribution function $E(x-a)$, where $a$ is a constant and $E(\xi)$ is the unity distribution, i.e. equal to $0, I$ depending on $\xi$ being negative or non-negative respectively. Further, Esscher has deduced a relation for the distribution functions defining a Poisson process with such a change distribution, implying that the distribution function for a fixed value of $t$ can be expressed as a convolution of a number of Bessel functions equal to the number of exponential terms in the change distribution with a distribution function based on the term $E(x-a)$. This relation is consistent with Almer's results quoted in the previous section, Esscher has also deduced a similar relation for the Polya process with a somewhat modified change distribution.

9. In the sequel, it will always be assumed that $V(x)$ is of the following form, in agreement with Esscher's suggestion.

$$
\begin{array}{r}
{ }_{b} V_{s}(x ; \beta)=B_{0} E(x-b)+\sum_{\rho=1} A_{\rho}\left(\mathrm{I}-e^{-\beta_{\mathrm{p}} x}\right) ; \beta=\left\{\beta_{\mathrm{p}}>0, \rho=\right. \\
=\mathrm{I}, 2 \ldots \mathrm{s}\}, b \geq 0
\end{array}
$$


If $b=\mathrm{o}$, so is $B_{0}$. By definition $B_{0}+\dot{\sum}_{\mathrm{p}-1} A_{\mathrm{p}}=\mathrm{x}$. The function is assumed to be normalised so that its mean $B_{0} b+\underset{p-1}{\dot{\sum}}\left(A_{f} / \beta_{\rho}\right)=\mathrm{I}$.

It is easily seen, that if $c x$ is substituted for $x$ in (IO) the change distribution can be written in the form

with the mean $\mathbf{I}$.

$$
{ }_{a} V_{s}(c x)=B_{0} E(c x-a)+\sum_{\rho-1} A_{\rho}\left(I-e^{-\alpha_{\rho} c x}\right) ; \alpha_{\rho}=\frac{\beta_{\rho}}{c}, a=c b
$$

The characteristic function of ${ }_{b} V_{s}(x),{ }_{b} z_{s}(u), u$ being an entirely imaginary variable, can be written

$$
b z_{s}(u)=B_{0} e^{b u}+\sum_{\rho=1} A_{\rho} h_{\beta_{\rho}, 1}(u),
$$

where we have introduced $b_{\beta, p}(u)=e^{b u}(\mathrm{I}-u / \beta)^{-p}$ which corresponds to the distribution function ${ }_{b} H_{1}(x ; p, \beta)$ defined by a Pearson type III density function represented by a curve beginning at $b \geq 0$. Let, further, the function ${ }_{a} H_{s}\left(x ; l_{1}, l_{2} \ldots l_{s}, \alpha_{1}, \alpha_{2} \ldots \alpha_{s}\right)$ be the convolution of ${ }_{s} H_{1}\left(x ; l_{1}, \alpha_{1}\right)$ with $(s-\mathrm{I})$ components in the form ${ }_{0} H_{1}\left(x ; l_{\rho}, \alpha_{\rho}\right)$ for $\rho=2,3 \ldots .$.

Let $i_{0}$ be the largest integer less than $x / a$ and let $A_{0}$ represent a modification of $B_{0}$ such that $a z_{s}^{i}(0)$ will be equal to I. Then, $a z_{2}^{i}(u)$ for $I<i<i_{0}$ can be written in the following form, derived by the iterated use of Newton's binomial formula.

$$
a_{2}^{z^{i}}(u)=\sum_{i=0}^{i} \sum_{1=0}^{i-l}\left(\begin{array}{l}
i \\
l
\end{array}\right)\left(\begin{array}{c}
i-l \\
j
\end{array}\right) A_{0}^{l} A_{1}^{i-j-l} A_{2}^{j} e^{l a u}{ }_{0} h_{\alpha_{1}, i-j-l}(u)_{0} h_{\alpha_{2}, j}(u)
$$

for $i>i_{0}, a z_{2}^{i}(u)$ is in the form of (II) with truncation of the first sum at $i_{0}$. By definition the conversion of (II) leads to an expression for $V^{i *}(x)$ in the same form with the substitution of

$$
{ }_{l a} H_{2}\left(x ; i-j-l, j, \alpha_{1}, \alpha_{2}\right) \text { for } e^{i a n}{ }_{0} h_{\alpha_{1}, i-j-l}(u){ }_{0} h_{\alpha_{2}, j}(u) .
$$

Io. Let the distribution function of $\bar{X}(t)$, as defined by (9), with the insertion of (Io) be denoted ${ }_{b \bar{X}} F_{s}(x, t)$, where the upper index 
$\varkappa$ shall be added, if $\bar{P}_{i}(t)$, particularly, is defined by a Hofmann probability ${ }_{\bar{q}}^{(x)} \bar{\pi}_{i}(t)$. Let, further, $Q_{s}(t)$ denote the necessary correction for the term for $i=0$, if this term should differ from $\bar{P}_{\mathbf{0}}(t)$.

$a \bar{X} F_{2}(x, t)$ can by (9) and (Io) be expressed in the form

$$
Q_{2}(t)+\sum_{i=0}^{i} \sum_{i=0}^{i}+\sum_{i=i_{0}+1}^{\infty} \sum_{i=0}^{i_{0}}=Q_{2}(t)+\sum_{i=0}^{i_{0}}\left(\sum_{i=1}^{i_{0}}+\sum_{i=i_{0}+1}^{\infty}\right)
$$

By the introduction of a new variable of summation in two stages, first by replacing $i$ with $i+l$, and, after reversing the order of the summation over $i$ and $j$, by replacing $i$ with $i+j$, the following expression is obtained for $a \bar{X}^{F_{2}}(x, t)$, where $(-t / c \bar{q})^{i+j+l} \bar{P}_{0}^{(i+j+l)}(t / c \bar{q})$ has been inserted for $(i+j+l) ! \bar{P}_{i+j+l}(t / c \bar{q})$ according to the identity given in (2).

$$
\begin{array}{r}
a \bar{X} F_{\mathbf{2}}(x, t)=Q_{\mathbf{2}}(t)+\sum_{i=0}^{b_{0}}\left(\frac{A_{0} t}{c \bar{q}}\right)^{l} \frac{\mathrm{I}}{l !} \sum_{i=0}^{\infty}\left(\frac{A_{1} t}{c \bar{q}}\right)^{i} \frac{\mathrm{I}}{i !} \sum_{j=0}^{\infty}\left(\frac{A_{2} t}{c \bar{q}}\right)^{j} \frac{\mathrm{I}}{j !}(-\mathrm{I})^{i+j+l} \times \\
\times \bar{P}_{0}^{(i+j+l)}(t / c \bar{q})_{l a} H_{2}\left(x, i, j, \alpha_{1} \alpha_{2}\right)
\end{array}
$$

By an analogous deduction the relation (I2) is extended to $\mathrm{s}=3$, which leads to a quadruple sum similar to the triple sum in (I2). By mathematical induction this result is extended to $s=4,5 \ldots$, the result in each case containing a $(s+\mathrm{I})$ - tuple sum.

II. In the particular cases, where $\bar{P}_{i}(t)$ are defined either by a Poisson probability distribution or a Polya probability distribution, i.e. by Hofmann probabilities with $x=0$ or I respectively, the distribution functions of $\bar{X}(t)$ can be written in the following comprehensive form.

$$
\stackrel{(x)}{a \bar{X}} F(c x, t)={ }^{(x)} Q_{s}(t)+\sum_{t_{0}=0}^{b_{0}} \frac{(x) t_{0}^{l_{0}}}{l_{0} !}{ }_{l_{0} a}^{(x)} G_{s}\left(c x ; \alpha_{1}, \alpha_{2}, \ldots \alpha_{s}\right),
$$

where ${ }^{(x)} Q_{s}(t)=\left\{\begin{array}{l}0 \text { for } s=2 \\ { }^{(x)} \bar{P}_{0}(t / c \bar{q})\left[I-{ }^{(x)} T_{s}\right] \text { for } s>2,\end{array}\right.$

and ${ }^{(x)} T_{s}$ will be defined here below in section $\mathrm{I}_{4}$. 


$$
\begin{aligned}
& { }_{l_{0} a}^{(x)} G_{s}\left(c x ; \alpha_{1}, \alpha_{2}, \ldots \alpha_{s}\right)=\sum_{l_{1}=0}^{\infty} \frac{t_{1}^{(x)} t_{1}^{l_{1}}}{l_{1} !} \sum_{l_{2}=0}^{\infty} \frac{{ }^{(x)} t_{2}^{l_{2}}}{l_{2} !} \ldots \sum_{l_{1}=0}^{\infty} \frac{{ }^{(x)} t_{s}^{l_{s}}}{l_{s} !}{ }^{(x)} \bar{P}_{0}(t / c \bar{q})(\bar{q})_{L}^{x} \times \\
& \times{ }_{a} H_{s}\left(c x ; l_{1}, l_{2} \ldots l_{s}, \alpha_{1} \alpha_{2} \ldots \alpha_{s}\right) \\
& { }^{(x)} t_{\rho}=A_{p}(t / c)(t / c+\bar{q})^{-x} \text { for } p=\mathrm{I}, 2 \ldots \mathrm{s}, x=0 \text {, I and for } p=0 \text {; if } s=2 \text {. }
\end{aligned}
$$

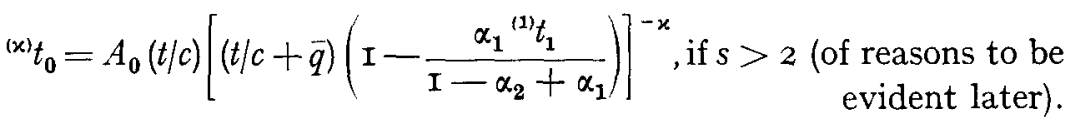

$$
\begin{aligned}
& { }^{(x)} \bar{P}_{0}(t / c \bar{q})=\left\{\begin{array}{l}
e^{-t / c}=e^{-\left(A_{0}+A_{1}+\cdots A_{0}\right) t / c} \text { for } x=0 \\
(\mathrm{I}+t / c \bar{q})^{-\bar{q}}=(\mathrm{I}+t / c \bar{q})^{-\left(A_{0}+A_{1}+\cdots A_{s}\right) \bar{q}} \text { for } x=\mathrm{I}
\end{array}\right. \\
& L=\sum_{\rho=0} l_{\rho} ;(\bar{q})_{L}=\bar{q}(\bar{q}+\mathrm{I}) \ldots(\bar{q}+L-\mathrm{I}) \text { for } L>0,(\bar{q})_{0}=\mathrm{I} \text {. }
\end{aligned}
$$

$A_{0}$ is defined by the assumptions that, for $x=0,\left(A_{0} t / c\right)^{l_{0}} e^{-A_{0} t / c / l_{0} !}$ and, for $x=\mathrm{I},\left[A_{0}(t / c)(t / c+\bar{q})^{-1}\right]_{0}^{l_{0}}[\mathrm{I}+t / c \bar{q}]^{-A_{0} \bar{y}} / l_{0} !$ represent discontinous, truncated probability distributions of the integer $l_{0}$, which cannot assume values greater than $i_{0}$, defined in section 9 as the greatest integer less than $c x / a$.

I2. The principal solution of Kummer's differential equation is called Kummer's function, here designated by $C(q, p+q ; x)$ for all real and complex values of $p, q, x$, excluding $p+q$ equal to zero or to a negative integer; it belongs to the class of confluent hypergeometric functions defined as the general solution of Kummer's equation. This function can be expressed by an absolutely convergent series given in (r4a) below (cf. Slater, Ig6o, I.I.8). It is connected with several well-known functions such as the $I^{\text {st }}$ kind Bessel function of the order of $q-\mathrm{I} / 2$, here designated by $\mathbf{I}_{q-1^{12}}(x)$, by Kummer's $2^{\text {nd }}$ theorem, and as the general incomplete gamma-function, here designated by $\gamma(q, x)$, the relations to these functions are given in ( $\mathrm{r}$ b), (I $4 \mathrm{c})$ respectively here below (Slater, r.8.1. - I.8.3, 5.6.2 and 5.6.4). A relation, implying a transformation of $C(q, p+q ; x)$, which will be used in the sequel, is asserted in Kummer's $\mathrm{I}^{\text {st }}$ theorem and is given in (I4d) here below (Slater, I.4.I). If the real parts of $q$ and of $p+q$ both are $>0$, the Kummer function may be expressed in terms of certain 
integrals given in (I4e), (I4f) here below (Slater, 5.6.9 and 3.I.2). The notation $(q)_{v}$ has been defined under ( $\left.\mathrm{I} 3\right)$.

$$
\begin{aligned}
& C(q, p+q ; x)=\sum_{\nu=0}^{\infty} \frac{(q)_{\nu} x^{\nu}}{(p+q)_{\nu} \nu !} \\
& I_{q-1 / 2}(x / 2)=\sum_{\nu=0}^{\infty} \frac{(x / 4)^{2 v+q-1 / 2}}{\Gamma(q+\mathrm{I} / 2+\nu) \nu !}=\frac{e^{-x / 2}(x / 4)^{q-1 / 2}}{\Gamma(q+\mathrm{I} / 2)} C(q ; 2 q ; x) \\
& \gamma(q, x)=\frac{e^{-x}}{q} \sum_{\nu=0}^{\infty} \frac{x^{\nu+q}}{(q+\mathrm{I})_{\nu}}=\frac{x^{q}}{q} C(q, \mathrm{I}+q ;-x) \\
& C(q, p+q ; x)=e^{x} C(p, p+q ;-x) \\
& C(q, \mathrm{I}+q ;-x)=q x^{-q} \int_{0}^{x} v^{q-1} e^{-v} d v, R l q>0 \\
& C(q, p+q ; x)=\frac{\Gamma(p+q)}{\Gamma(p) \Gamma(q)} \int_{0}^{1} e^{x t} u^{q-1}(\mathrm{I}-u)^{p-1} \mathrm{du}, R l p>0,
\end{aligned}
$$

I3. In the deduction of expressions for ${ }_{a} H_{s}(c x)$, it is assumed that $\alpha_{1}<\alpha_{2} \ldots \alpha_{s}$. If this assumption should not be true the definition of ${ }_{a} H_{s}(x)$ is such that $\left(\alpha_{1}, l_{1}\right),\left(\alpha_{2}, l_{2}\right) \ldots\left(\alpha_{s}, l_{s}\right)$ may be permutated, without change of ${ }_{a} H_{s}(x)$; this implies that the assumption does not restrict the generality of our deductions.

By definition we have

$$
{ }_{a} H_{2}\left(c x ; p, q, \alpha_{1}, \alpha_{2}\right)=\int_{a}^{c x} \frac{\alpha_{1}^{p} \alpha_{2}^{q}}{\Gamma(p) \Gamma(q)} d v \int_{0}^{e^{-a}} \begin{array}{r}
e^{-\alpha_{1}(v-a-z)-\alpha_{2} y}(v-a-z)^{p-1} \\
z^{q-1} d z
\end{array}
$$

By introducing the new variables of integration $u, \xi$ by the substitution of $u \xi$ for $z$ and of $\xi$ for $v-a$, the inner integral of (15) becomes equal to

$$
\int_{0}^{1} e^{-\delta_{1} \xi u} u^{q-1}(\mathrm{x}-u)^{p-1} d u, \delta_{1}=\alpha_{2}-\alpha_{1}
$$


which by (I4f) can be expressed in terms of $C\left(q, p+q,-\delta_{1} \xi\right)$. By the transformation of the Kummer function by (I4d)-equivalent to the aforesaid permutation of the parameters-the double integral can by (I4a) be written in the following form, where, on account of the absolute convergency of (I4a) and of the integral over the Pearson type III density, the order of summation and integration has been reverted.

$$
\sum_{\rho_{2}=0}^{\infty} \frac{\Gamma\left(p+\rho_{2}\right) \alpha_{1}^{p} \delta_{1}^{\rho_{2}}}{\Gamma(p) \alpha_{2}^{\rho_{2}+p} \rho_{2} !} \int_{0}^{c x-a} \frac{\alpha_{2}^{p+q+\rho_{2}} \xi^{p+q+\rho_{2}-1}}{\Gamma\left(p+q+\rho_{2}\right)} e^{-\alpha_{2} \xi} d \xi,
$$

which, with respect to the definition of ${ }_{a} H_{2}(x)$, is defined for real values of the variable and of the parameters with $x \geq 0, a \geq 0$, $p>0, q>0, \alpha_{1}>0, \alpha_{2}>0$. In fact, (I6) is an expression for (I5), also if (I5) should have been defined for complex valued variable and parameters, provided that their real parts fulfil the same inequalities.

By the substitution of ${ }_{0} H_{2}\left(c x-a ; p+q+p_{2}, r, \alpha_{2}, \alpha_{3}\right)$ for the integral in (I6) and by an analogous deduction for the inserted form of ${ }_{0} H_{2}(c x-a)$ an expression for ${ }_{a} H_{3}\left(c x, p, q, \gamma, \alpha_{1}, \alpha_{2}, \alpha_{3}\right)$ is obtained, which is in the form of a weighted sum of (I6) with similar weight functions as those appearing in (I6). By mathematical induction the expression can be extended to $s=4,5 \ldots$ which leads to the following Theorem $\mathbf{I}$.

Theorem I. Let $0<\alpha_{1}<\alpha_{2} \ldots<\alpha_{s}$ be an increasing sequence of real parameters, $\lambda_{1}, \lambda_{2}, \ldots \lambda_{s}$ be a sequence of positive numbers, $b_{1}=a \geq 0$ and $b_{v}$ for $v \neq \mathrm{I}$ equal to zero. Let $x_{1}, x_{2} \ldots x_{v}, x_{v+1} \ldots x_{s}$ be a sequence of random variables, each of which being distributed with a distribution function in the form

$$
b_{\nu} H_{1}\left(x ; \lambda_{\nu}, \alpha_{v}\right)=\frac{\alpha_{\nu}^{\lambda_{\nu}}}{\Gamma\left(\lambda_{\nu}\right)} \int_{b_{v}}^{x}\left(\xi-b_{v}\right)^{\lambda_{\nu}-1} e^{-\alpha_{\nu}\left(\xi-b_{v}\right)} d \xi,
$$

and let the functions $f_{\rho_{v}}$ for each value of $\nu=2,3 \ldots s$ be defined by

$$
f_{\rho v}=\frac{\Gamma\left(\Lambda_{\nu-1}+R_{v}\right)}{\Gamma\left(\Lambda_{v-1}+R_{v-1}\right) \rho_{\nu} !}\left(\frac{\alpha_{\nu}-\alpha_{\nu-1}}{\alpha_{\nu}}\right)^{\rho_{\nu}}\left(\frac{\alpha_{v-1}}{\alpha_{\nu}}\right)^{\Lambda_{v-1}+R_{v-1}},
$$


where $\Lambda_{\nu}=\sum_{r=1}^{\nu} \lambda_{r}, R_{v}=\sum_{r=1}^{\nu} \rho_{r}$,

Then, the distribution function of $x=x_{1}+x_{2} \ldots+x_{s}$ is given by the $(s-$ I)-tuple sum

$$
\sum_{\rho_{2}=0}^{\infty} f_{\rho_{2}} \sum_{\rho_{3}=0}^{\infty} f_{\rho_{s}} \ldots \sum_{\rho_{s}=0}^{\infty} f_{\rho_{s} a} H_{1}\left(x ; \rho_{s}+\Lambda_{s}, \alpha_{s}\right) .
$$

I4. The following expression for ${ }_{a} H_{1}(x ; p, \beta)$ in (I $\left.7 \mathrm{a}\right)$ is obtained by using the relations (I4C), (I4e). A particular case of this expression is the expansion of the distribution function of $\chi$-square, as commonly used in statistical tables (cf. e.g. Fisher and Yates (I938) p. I; Pearson and Hartley (I954), pp. I8-20). It has also been used with $\beta=I$ and $t-x$ substituted for $x-a$ for the deduction of the distribution functions defining a Poisson process with the change distribution defined by a single exponential term by Cramér (I955, p. 4I). The function ${ }_{a} \bar{H}_{s}\left(x ; l_{1}, l_{2} \ldots l_{s}, \alpha_{1}, \alpha_{2} \ldots \alpha_{s}\right)$ shall be defined by the integral

$$
\int_{0}^{x}(v-a) d_{v a} H_{s}\left(v ; l_{1}, l_{2} \ldots l_{s}, \alpha_{1}, \alpha_{2} \ldots \alpha_{s}\right) .
$$

An expression for ${ }_{a} H_{1}(x ; p, \beta)$ similar to (I $\left.7 \mathrm{a}\right)$ is easily obtained by this definition.

$$
\begin{aligned}
& { }_{a} H_{1}(x ; p, \beta)=\frac{I}{\Gamma(p)} \int_{0}^{\beta(x-a)} v^{p-1} e^{-v} d v=e^{-\beta(x-a)} \sum_{\nu=0}^{\infty} \frac{[\beta(x-a)]^{v+p}}{\Gamma(v+p+I)}(\text { I } 7 \mathrm{a}) \\
& \bar{a}_{1}(x ; p, \beta)=\frac{\mathrm{I}}{\beta \Gamma(p)} \int_{0}^{\beta(x-a)} v^{p} e^{-v} d v=\frac{p e^{-\beta(x-a)}}{\beta} \sum_{v=0}^{\infty} \frac{[\beta(x-a)]^{v+p+1}}{\Gamma(\nu+p+2)}(\mathrm{I} z \mathrm{~b})
\end{aligned}
$$

By definition, $x \geq 0, a \geq 0, p>0, \beta>0$ are real-valued. The deduction holds even, if these magnitudes are allowed to be complex provided that the same inequalities hold for the real parts. It shall be observed, however, that if the $3^{\text {rd }}$ membrum of (I7a) is taken as definition for ${ }_{a} H_{1}(x)$ we may extend the definition to $p=0$, in 
which case this membrum reduces to unity. Also ${ }_{a} \bar{H}_{1}(x)$ may be defined by the $3^{\text {rd }}$ membrum of $(\mathrm{I} 7 \mathrm{~b})$, for $p=0$ inclusive, in which case this membrum vanishes. By inserting the relation (I7a) in ${ }_{a}^{(0)} G_{1}\left(x, \alpha_{1}\right)$ according to its definition under (13) this function can be written in the form

$$
e^{-\alpha, \xi} \sum_{v=0}^{\infty}\left(\frac{\sqrt{\tau_{1} \xi}}{t_{1}}\right)^{\nu} I_{v}\left(2 \sqrt{\tau_{1} \xi}\right)
$$

where $\tau_{1}=\alpha_{1} A_{1} t ; \xi=x-a$ and $\mathrm{I}_{\mathrm{v}}(x)$ the $\mathrm{I}^{\text {st }}$ kind Bessel function defined in $(\mathrm{I} 4 \mathrm{~b})$. If $(\mathrm{I} 7 \mathrm{~b})$ is inserted for ${ }_{a} H_{1}(x)$ in the relation defining ${ }_{a}^{(0)} G_{1}(x)$, the function obtained being denoted with a bar, a similar expression for this function is obtained, namely,

$$
\frac{\mathrm{I}}{\alpha_{1}} \sum_{v=0}^{\infty}\left(\frac{\sqrt{\tau_{1} \xi}}{t_{1}}\right)^{v+2} \mathrm{I}_{v+2}\left(2 \sqrt{\tau_{1} \xi}\right)
$$

These relations lead to simple numerical calculation for $x=0$, $s=\mathrm{I}$; as, however, the corresponding expressions for $x=\mathrm{I}$ and for $s>\mathrm{I}$ are more complicated, the author refrains from following this line any further.

I5. If the expression for ${ }_{a} H_{2}\left(c x ; i, j ; \alpha_{1}, \alpha_{2}\right)$ for $i, j$ being positive integers according to Theorem $\mathrm{I}$ is inserted in the relation defining ${ }_{a}^{(0)} G_{2}(x)$, under (I3), and if the new variables of summation $\mu_{2}, r$ are introduced by the substitution of $\mu_{2}-i$ for $p_{2}$ and of $r-\mu_{2}$ for $j$ the function ${ }_{a}^{(0)} G_{2}\left(c x ; \alpha_{1}, \alpha_{2}\right)$ can be written as follows ${ }_{a}^{(0)} G_{2}\left(c x ; \alpha_{1}, \alpha_{2}\right)=\sum_{i=0}^{\infty} \sum_{\mu_{2}-i}^{\infty} \sum_{r=\mu_{2}}^{\infty}\left(\begin{array}{c}\mu_{2}-\mathrm{I} \\ i-\mathrm{I}\end{array}\right) \frac{\left({ }^{(0)} \tau_{1}^{i(0)} \tau_{2}-\mu_{2} \delta_{1}^{\mu_{2}-i} t_{2}^{r}\right.}{i !\left(r-\mu_{2}\right) !}{ }_{a} H_{1}\left(c x ; r, \alpha_{2}\right)$, where $\delta_{v}=\alpha_{v+1}-\alpha_{v} ;{ }^{(x)} \tau_{v}=\alpha_{v}{ }^{(\alpha)} t_{v}$.

By (I7a), (I8) can be expressed in the form of a quadruple sum. If in this sum the variable $j$ is reintroduced by substituing $j+\mu_{2}$ for $r$, the sum will be of the form

$$
\sum_{i=0}^{\infty} \sum_{\mu_{2}=-}^{\infty} \sum_{i=0}^{\infty} \sum_{-,+, w_{2}}^{\infty}
$$


As, independently of $c$, all the summations refer to an absolutely convergent series of terms, which follows from the definitions under (I3) and under (I8), the order of summations may be reversed by the identities

$$
\sum_{i=0}^{\infty} \sum_{\mu_{2}-i}^{\infty}=\sum_{\mu_{2}=0}^{\infty} \sum_{i=0}^{\mu_{2}} \text { and } \sum_{j=0}^{\infty} \sum_{r-i+\mu_{2}}^{\infty}=\sum_{\nu=\mu_{2}}^{\infty} \sum_{i=0}^{v},
$$

this leads to an expression for ${ }_{a}^{(0)} G_{2}(c x)$, where $c$ can be chosen equal to unity, in a form which is consistent with the general relations given here below. The relation is easily extended to ${ }_{a}^{(1)} G_{2}(x)$ by its definition under (13).

If in (I8) ${ }_{a} H_{2}\left(c x ; r, k, \alpha_{2}, \alpha_{3}\right)$ is substituted for ${ }_{a} H_{1}\left(c x ; r, \alpha_{2}\right)$ and if the expression obtained is multiplied by ${ }^{(0)} t_{3}^{k} / k !$ and summed over $k$ from $k=0$ to $k=\infty$, an expression for ${ }_{a}^{(0)} G_{3}\left(c x ; \alpha_{1}, \alpha_{2}, \alpha_{3}\right)$ is obtained. Inserting in this expression the expression for ${ }_{a} H_{2}\left(c x ; r, k, \alpha_{2}, \alpha_{3}\right)$ in accordance with the deduction of (18) and by using (I7a) a 6-tuple sum is obtained. If in this sum the variables $j, \mu_{2}$ and $k$ are reintroduced, an expression is obtained, which can be written as a product of a double sum and a quadruple sum in the form of ${ }_{a}^{(0)} G_{2}(c x)$. By the application of the same deduction to ${ }_{a}^{(1)} G_{3}(c x)$, it is found that a similar double sum appears in each term of the quadruple sum. For $x=0, I$ the double sum just mentioned can be written

$$
\sum_{i=0}^{\infty} \frac{\left[(\bar{q})_{i+j+k+l}\right]^{x}}{i !} \tau_{1}^{i} \sum_{\mu_{2}=0}^{\infty} \frac{(i)_{\rho_{2}} \delta_{1}^{\rho_{2}}}{\rho_{2} !}, x=0, \mathrm{I}
$$

If $\delta_{1}=\alpha_{2}-\alpha_{1}>0$ is $<I$, the inner sum of this expression converges to $\left(\mathrm{I}-\delta_{1}\right)^{-i}$ for each value of $i$, and, for $x=0$, the double sum reduces to $\exp \left[{ }^{(0)} \tau_{1} /\left(I-\delta_{1}\right)\right]$. If, in addition, $\alpha_{1} /\left(I-\delta_{1}\right)$ $<\mathrm{I}$, the double sum for $x=\mathrm{I}$ converges, as by definition ${ }^{(1)} \tau_{1}<\mathrm{I}$, to $\left[\mathrm{I}-{ }^{(1)} \tau_{1} /\left(\mathrm{I}-\delta_{1}\right)\right]^{-\bar{q}-j-k-l}$. If the change distribution ${ }_{b} V_{s}(x)$ has parameters $\beta_{\rho}$ such that these conditions are not fulfilled for $c=I$, it is always possible to choose $c$ as a constant, for $x=0,>$ $\operatorname{Max}\left(\beta_{\rho+1}-\beta_{\rho}\right)$, and, for $x=I,>\operatorname{Max}\left(\beta_{p+1}-\beta_{\rho}, \beta_{2}\right)$. By this choice $\delta_{\rho}<I$ for $\rho=I, 2 \ldots$ independently of $x$ and, for $x=I, \alpha_{1} /\left(I-\delta_{1}\right)$ $<$ I. Choosing $c$ in this way, the expression for ${ }_{a}^{(0)} G_{3}(c x)$ reduces 
to ${ }_{a}^{(0)} G_{2}\left(c x ; \alpha_{2}, \alpha_{3}\right)$ multiplied by an exponential factor and ${ }_{a}^{(1)} G_{3}(c x)$ to a function in the same form, with the substitution of a power of $I-{ }^{(1)} \tau_{1} /\left(I-\delta_{1}\right)$ for the exponential factor and of ${ }_{3} \theta_{\rho}=$ ${ }^{(1)} \tau_{\rho} /\left[I-{ }^{(1)} \tau_{1} /\left(I-\delta_{1}\right)\right]$ for ${ }^{(0)} t_{\rho}, p=2,3$ respectively. For $x=0$ the result may in an analogous way be extended to an expression for ${ }_{a}^{(0)} G_{4}(c x)$, and, by mathematical induction, to ${ }_{a}^{(0)} G_{s}(c x), s>4$. And as ${ }_{a}^{(1)} G_{3}(c x)$ is in the form of ${ }_{a}^{(0)} G_{3}(c x)$ the extension to ${ }_{a}^{(1)} G_{s}(c x), s>3$ immediately follows. By ( $\mathrm{I} 7 \mathrm{~b})$ similar relations for ${ }_{a}^{(x)} \bar{G}_{s}(c x)$, defined at the end of the previous section, are obtained. The deductions given here above in this section lead to the following theorem.

Theorem 2. Let for $x=0, \mathrm{I}$ and

$$
\begin{aligned}
& \text { for } s=\mathrm{I}, 2 \text {; } \\
& \text { and for } s>2 \\
& { }_{s}^{(x)} \theta_{p}=\alpha_{p} A_{p}(t / c)(t / c+\bar{q})^{-x} ; \quad \stackrel{(x)}{s} \theta_{p}=\alpha_{p} A_{p}(t / c)^{x} \\
& {\left[(t / c+q)\left(\mathrm{I}-{ }_{1}^{(1)} \theta_{1} /\left(\mathrm{I}-\delta_{1}\right)\right)\right]^{-x} ;} \\
& \rho=I, 2 \ldots s, \\
& { }_{s}^{(x)} U_{v}=\sum_{j=0}^{\nu} \frac{{ }_{s} \theta_{s}^{j}}{\alpha_{s}^{j} j !}(\bar{q})_{(s-1) i+j+l_{0}}^{x} ; \quad{ }_{s}^{(x)} U_{v}=\sum_{i=0}^{\nu} \frac{{ }^{s} \theta_{s}^{j}}{\alpha_{s}^{j} j !} ; \\
& { }_{s}^{(x)} T=\mathbf{I} \\
& { }_{s}^{(x)} T=\left[\left(\mathrm{I}-{ }_{1}^{(1)} \theta_{1} /\left(\mathrm{I}-\delta_{1}\right)\right)^{\bar{\varphi}} e^{(1) \theta_{1} /\left(1-\delta_{1}\right)}\right]^{-x} \times \\
& \times \exp \cdot \sum_{\rho-1}^{i-2} \frac{{ }^{(x)} \theta_{p}}{\mathrm{I}-\delta_{\rho}} \\
& { }_{s}^{(x)} \bar{U}^{\nu}={ }_{s}^{(x)} U_{\nu-1}+\mu_{s}{ }_{s}^{(x)} U_{\nu} \quad ; \quad{ }^{(x)} S_{\mu}=\sum_{\mu=0}^{\infty} \frac{{ }^{(x)} \theta_{s-1}^{2} \delta_{s-1}^{-i}}{i !(i-1) !(\mu-i) !} ; \\
& { }^{(x)} P_{0}(t)=\left\{\begin{array}{l}
e^{-\bar{q} t} \text { for } x=0 \\
(\mathrm{I}+t)^{-\bar{q}} \text { for } x=\mathrm{I}
\end{array}\right.
\end{aligned}
$$

and $\xi=x-b$, then

the function ${ }_{a} G_{s}(c x)$ appearing in (I3), which defines the normalized distribution functions of $\bar{X}(t)$, for $x=0$, attached to a Poisson process with the change distribution ${ }_{a} V_{s}(c x)$ as defined under (Io) and, for $x=\mathrm{I}$, to a Polya process with the same change distribution can be written in the following form for $s>\mathrm{I}, x=0, \mathrm{I}$. 


$$
\begin{aligned}
{ }_{a}^{(x)} G_{s}\left(c x ; \alpha_{1}, \alpha_{2} \ldots \alpha_{s}\right)={ }^{(\alpha)} \bar{P}_{0}(t / c \bar{q}) e^{-\alpha s c \xi(x)} T & \sum_{\mu_{s}=0}^{\infty} \frac{\delta_{s-1}^{\mu_{s}} \Gamma\left(\mu_{s}\right)}{\alpha_{s}^{\mu_{s}}}{ }^{(x)} S_{\mu_{s}} \times \\
& \times \sum_{\nu=\mu_{v}}^{\infty} \frac{\left(\alpha_{s} c \xi\right)^{\nu}}{\nu !}{ }^{(x)} U_{v}
\end{aligned}
$$

and the function $a \bar{G}_{s}(c x)$ defined at the end of the previous section for $s>\mathrm{I}, x=0, \mathrm{I}$.

$$
\begin{aligned}
& { }_{a}^{(\alpha)} \bar{G}_{s}\left(c x ; \alpha_{1}, \alpha_{2} \ldots \alpha_{s}\right)={ }^{(x)} \bar{P}_{0}(t / c \bar{q}) \frac{e^{-\alpha \alpha c \xi}}{\alpha_{s}}{ }_{s}^{(x)} T \sum_{\mu_{s}=0}^{\infty} \frac{\delta_{s-1}^{\mu_{s}} \Gamma\left(\mu_{s}\right)}{\alpha_{s}^{\mu_{s}}} \times \\
& \times{ }^{(x)} S_{\mu_{\varepsilon}} \sum_{\nu=\mu_{q}}^{\infty} \frac{\left(\alpha_{s} c \xi\right)^{\nu+1}}{(u+\mathrm{I}) !}{ }^{(x)} \bar{U}_{\nu}
\end{aligned}
$$

If in (rga), (Igb) $\sum_{\mu_{0}-0}^{\infty}$ is replaced by its term for $\mu_{s}=0$ the relations hold for $s=\mathrm{I}$.

With the purpose of giving (Iga), (rgb) in forms apt for the numerical calculation with an electronic computer, the relations are transformed to the following expressions for $s>2$ and for $s=\mathbf{I}$.

Starting with the simpler case, we denote the $\nu^{\text {th }}$ term of the sums appearing in the expression (Iga) and (Igb) for $s=\mathrm{I}$ by ${ }_{1}^{(x)} K_{v},{ }_{1}^{(x)} \bar{K}_{v}$ respectively. Then,

$$
\begin{aligned}
& { }_{1}^{(\alpha)} K_{\nu}=\frac{\alpha_{s} c \xi}{\nu}\left[{ }_{1}^{(x)} K_{\nu-1}+{ }_{1}^{(x)} N_{v}\right] ;{ }_{1}^{(x)} N_{v}=\frac{{ }^{(x)} \tau_{1} c \xi}{\nu(\nu-I)}\left(\bar{q}+\nu+l_{0}-\mathrm{I}\right)^{x} \cdot{ }_{1}^{(x)} N_{\nu-1}= \\
& ={ }_{1}^{(x)} g_{y}{ }_{1}^{(x)} N_{v-1} \text {, say. } \\
& { }_{1}^{(x)} \bar{K}_{v}=\frac{\alpha_{s} c \xi}{\nu+I}\left[{ }_{1}^{(x)} \bar{K}_{v-1}+{ }_{1}^{(x)} \bar{N}_{v}\right] ;{ }_{1}^{(x)} \bar{N}_{v}=\frac{\left.{ }^{(x)}\right)_{1} c \xi}{v(v-I)}\left(\bar{q}+v+l_{0}-2\right)^{x} \cdot{ }_{1}^{(x)} \bar{N}_{v-1}= \\
& =\left[{ }_{1}^{(x)} g_{v}-x_{1}^{(0)} g_{v}\right]{ }_{1}^{(x)} \bar{N}_{v-1}
\end{aligned}
$$

Denote the $\mu^{\text {th }}$ term of the sums appearing in the expressions (Iga) and (Igb) for $s>\mathrm{I}$ by ${ }^{(x)} S_{\mu}{ }_{s}^{(x)} L_{\mu}$ and ${ }^{(x)} S_{\mu}\left({ }_{s}^{(x)} \bar{L}_{\mu}+{ }_{s}{ }_{s} \bar{K}_{\mu}\right)$ respectively. Then, for a fixed value of $s>2$, we have, simplifying the notations by writing $\mu, \theta_{\rho}$ for $\mu_{s},{ }_{s}^{(x)} \theta_{\rho}$ respectively, the following recurrence relations for ${ }_{s}^{(x)} L_{\mu},{ }_{s s}^{(x)} \bar{L}_{j \mu}$ and ${ }_{s}^{(x)} \bar{K}_{; \mu}$. 


$$
\begin{aligned}
& { }_{s}^{(x)} L_{\mu}=\frac{\delta_{s-1}(\mu-I)}{\alpha_{s}}{ }_{s}^{(x)} L_{\mu-1}-{ }_{s}^{(x)} M_{\mu} ; \\
& { }_{s}^{(x)} M_{\mu}=\delta_{s-1} c \xi\left({ }_{s}^{(x)} M_{\mu-1}+{ }_{s}^{(x)} N_{\mu}\right) ;{ }_{s}^{(x)} N_{\mu}=\frac{\theta_{s} \delta_{s-1} c \xi}{\alpha_{s}(\mu-I)}{ }_{s}^{(x)} N_{\mu-1} . \\
& { }_{s}^{(x)} \bar{L}_{\mu}=\frac{\delta_{s-1}(\mu-I)}{\alpha_{s}}{ }_{s}^{(x)} \bar{L}_{\mu-1}-{ }_{s}^{(x)} \bar{M}_{\mu} ; \\
& { }_{s}^{(x)} \bar{M}_{\mu}=\frac{\delta_{s-1} c \xi(\mu-I)}{\mu}\left({ }_{s}^{(x)} \bar{M}_{\mu-1}+{ }_{s}^{(x)} \bar{N}_{\mu}\right) ;{ }_{s}^{(x)} \bar{N}_{\mu}=\frac{\theta_{s} \delta_{s-1} c \xi}{\alpha_{s}(\mu-2)}{ }_{s}^{(x)} \bar{N}_{\mu-1} ; \\
& { }_{s}^{(x)} \bar{K}_{\mu}=\frac{\delta_{s-1} \mu}{\alpha_{s}}{ }_{s}^{(x)} \bar{K}_{\mu-1}-\alpha_{s} c \xi{ }_{s}^{(x)} M_{\mu} .
\end{aligned}
$$

For the calculation of ${ }^{(x)} S_{\mu}$ we write $\sum_{\mu=0}^{\infty} \varphi_{\mu-i}{ }^{(x)} \chi_{i}$ for ${ }^{(x)} S_{\mu}$ and, then, ${ }^{(x)} \chi_{i}=\frac{\theta_{s-1}}{\delta_{s-1} i(i-\mathbf{I})}{ }^{(x)} \chi_{i-1} ; \varphi_{\lambda}=\frac{I}{\lambda} \varphi_{\lambda-1}$.

I6. The main part of the calculation for $s=\mathrm{I}$ of (19a), (Igb) consists in the successive computation of (20a), (20b) from the initial values, $\nu=I$, and the successive accumulation of the results. Provided that these calculations are performed simultaneously for (20a), (2ob) and for $x=0, \mathrm{I}$, this implies, for each given vector $\alpha_{1} \xi, \tau_{1} \xi$ and for each value of $\nu, I_{3}$ "multiplications" (the word here taken to mean multiplication or division) and $\mathrm{I} 6$ additions. The number of values of $v$ needed for the computation depends on the precision wanted and on the order of magnitude of $\alpha_{1} \xi$, $\tau_{1} \xi$. In order to obtain a basis of comparison for the computation of (I9a), (Igb) for higher values of $s$ of the time required on an electronic computer available, a "computational unit" shall be defined as the time needed for the calculation of (2oa), (2ob) for $s=\mathrm{I}$, for each given vector $\alpha_{1} \xi, \tau_{1} \xi$ and for each value of $v$. Thus, the calculation of a sum of $n$ terms will require $n$ computational units, if $s=\mathrm{I}$.

If (2Ic) has been precalculated, (2Ia), (2Ib) imply for $x=0$ and I, 24 "multiplications" and 22 additions, which corresponds to about 2 computational units for a given vector, $c \xi,{ }^{(x)} t_{1},{ }^{(x)} t_{2}$, $\alpha_{s}, \alpha_{s-1}$ and for each value of $v$. The computation of (2Ic) can be 
performed as follows, let $m$ be the highest value of $i$ needed for the precision wanted, thus, $m$ depends on $\theta_{s-1} / \delta_{s-1} .{ }^{(x)} \chi_{i}$ is, then, calculated for each value of $2,3 \ldots \max (m)$ and each term is stored, without accumulation, then, the arguments of the values obtained are changed by the substitution of $\lambda$ for $m-i . \varphi_{\lambda}$ is calculated by (2IC) for each value of $\lambda$ and each term stored, without accumulation. Thereinafter, ${ }^{(x)} \chi_{m-\lambda} \varphi_{\lambda}$ is calculated for each value of $\lambda$ and $m$ with successive accumulation of the results, so that ${ }^{(x)} S_{\mu}$ is obtained for different values of $\mu$. The number $m$ of terms needed does not depend on $\xi$; a rough evaluation has shown that we might expect $m$ for a given value of $\theta_{s-1} / \delta_{s-1}$ to be of the order of $n / 2$, where $n$ is the number of terms needed for calculating (2Ia), (2Ib).

Suppose that we want to calculate (Iga), (Igb) for a fixed value of $s>2$, for $x=0, \mathrm{I}$, for a fixed vector of the parameters in ${ }_{b} V_{s}(x)$, and for $t=100$ combined with 5 values of $c \xi$, for $t=500$ combined with I 2 values of $c \xi$, and for $t=1000$ combined with I3 values of $c \xi$. Let us, further, assume that for each $t$ the number of values needed for the calculation of (2Ia), $(2 \mathrm{Ib})$, i.e. $n$, is on an average equal to r. $6 t$. The calculation of ${ }^{(x)} S_{\mu}$ for each fixed vector of ${ }^{(x)} \theta_{s-1} / \delta_{s-1}, x=0$ and $\mathrm{I}$, will, then require $\frac{1}{13}\left(m_{1}^{2}+\right.$ $\left.m_{2}^{2}+m_{3}^{2}+4^{2} m_{3}\right)$, where $m_{1}, m_{2}, m_{3}$ are values of $m$ determined by $t=100,500$, and $\mathrm{I}$ ooo respectively. By the assumptions made, this corresponds to about $227 \bar{n}$, where $\bar{n}=\frac{1.6}{3}(\mathrm{r} 000+500+$ $\mathrm{IO0})=820$. The computation of $(2 \mathrm{Ia}),(2 \mathrm{Ib})$ requires for the 30 values of the vector $(t, c \xi)$ and for a given vector of $\alpha_{s}, \alpha_{s-1}, A_{s}$, $A_{s-1}$ about $60 \tilde{n}$ computational units. If, further, 40000 computational units are allowed for the administration of the calculation of (2Ia) - (2IC) we should reach a total time for the calculation of (Iga), (Igb) for $s>2$ of 275000 computational units which, if 50 ooo such units should correspond to one hour, should be equivalent to $5 \mathrm{I} / 2$ hours for the series assumed. The corresponding figure for $s=\mathrm{I}$ should be 25 minutes on similar assumptions.

The corresponding relations for $s=2, x=I$ lead to materially more computation work because of the implications in ${ }_{2}^{(1)} U_{v}$. These implications have for $s>2$ been eliminated by the limit passage for the double sum discussed in the deduction of ${ }_{a}^{(x)} G_{3}(c x)$.

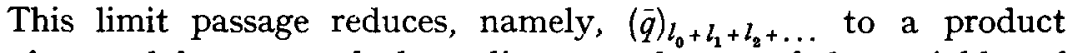
of several factors, each depending on only one of the variables of 
summation. For $s=2, x=0$ similar relations hold as those derived for $s>2, x=0, I$.

The numerical experience shows, that the number of terms in the sum appearing in the expressions for ${ }_{a}^{(\underline{x})} \bar{X} \cdot(c x, t)$ according to (13) is very restricted. The computation work implied in (13), if the functions $i_{0}^{(x)} G_{s}\left(c x ; \alpha_{1}, \alpha_{2} \ldots \alpha_{s}\right)$ have been precalculated, is, therefore, negligible. The series of calculations suggested here above, will give a fairly good mapping of the distribution functions defined by (I3). The corresponding stop loss risk premiums are obtained, with negligible extra work, from ${ }_{l_{0} a}^{(\alpha)} \bar{G}_{s}\left(c x ; \alpha_{1}, \alpha_{2} \ldots \alpha_{s}\right)$ as determined in the same series, by using the following formula

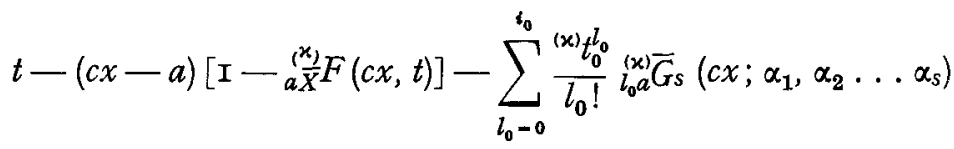

where ${ }^{\left({ }^{x}\right)} t_{0}$ for $s>2$ have been defined under (I3).

I7. Esscher has made a remark with respect to the relation (I) as a definition for the wide sense compound Poisson process, implying that the Poisson expression in the integrand of (I) ought to be dependent not only on time but also on the magnitude of the population for which the compound process is studied, while $U(v ; t)$ ought to be independent of this magnitude. On the other hand, it is possible that $U(v, t)$, at least in certain applications, depends not only on time but also on another parameter, which determines the distribution of $v$. - By choosing $U(v, t)$ in a suitable form to account for such circumstances, the approach in (I) will be valid also with regard to Esscher's remark. In the particular cases introduced in the deduction of (7), (8) the generality has been restricted by the assumption that the means of the two processes involved in (7), (8) before normalization are equal to $q \tau, \bar{q} \tau$ respectively for each given value of $\tau$. This implies, in fact, that the magnitude of the population involved in each of the processes is independent of time. The generalization of (7), (8) to include also non-stationary populations may be easily made on particular assumptions with respect to their variation with time. This will, however, not be done in the present paper.

I8. If in $(7)_{b} V_{s}(x)$ is inserted for $V(x)$, and by using, for $x=\mathrm{I}$, 


$$
{ }_{a Y}^{(0)} F_{s}(c x, t)=\sum_{m=0}^{\infty} P_{m}\left(\sqrt{t} / r_{0}\right){ }_{a}^{(0)} F_{s}\left(c x, \frac{m \sqrt{t}}{r_{0}}\right)
$$

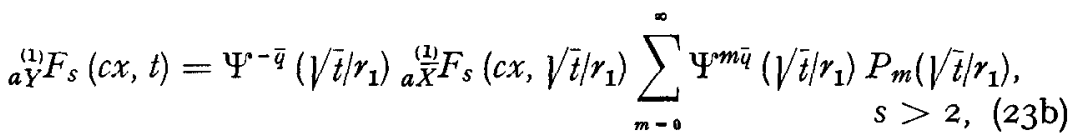
where $\Psi(t)=\left[\left(\mathrm{I}-\frac{A_{1} \alpha_{1} t}{\mathrm{I}-\delta_{1}}\right)(\mathrm{I}+t)\right]^{-1}$.

If, further, it is assumed that $P_{m}(t), q \pi_{m}(t)$ are defined by Poisson and Polya probabilities and observing, that by assumption in the deduction of $(7),(8)$ it has been assumed that their means shall be equal to $q \tau$, we obtain in these cases

$P_{m}\left(\sqrt{t} / r_{0}\right)={ }_{q} \pi_{m}\left(\sqrt{t} / r_{0}\right)=\left(q \sqrt{t} / r_{0}\right)^{m} e^{-q \mid \bar{t} / r_{0}} / m !$ in the $\mathrm{I}^{\text {st }}$ case

$$
P_{m}\left(\sqrt{t} / r_{1}\right)={ }_{q} \pi_{m}\left(\sqrt{t} / r_{1}\right)=\left(\begin{array}{c}
-q \\
m
\end{array}\right) \frac{\left(-\sqrt{t} / r_{1}\right)^{m}}{\left(\mathrm{I}+\sqrt{t} / r_{1}\right)^{m+q}} \text { in the } 2^{\text {nd }} \text { case }
$$

By combining each of (23a) and (23b) with both (24a) and (24b) we have to deal with 4 different distribution functions of $Y(t)$.

The numerical calculation of (23a) with the insertion of (24a), (24b) implies the calculation of ${ }_{a}{ }_{a}^{(0)} F\left(c x, \frac{m \sqrt{\bar{t}}}{r_{0}}\right)$ for each value of $m=0, \mathrm{I} \ldots$ to $m_{t}$ say, where $m_{t}$ shall be determined by the precision wanted and by the order of magnitude of the $m^{\text {th }}$ term of the sum appearing in (23a). A numerical estimation of this order for the case where (24a) is inserted in (23a) has been made in the following way. The probability of $i$ changes in $Y(t)$, while the parameter passes from $o$ to a fixed value $t$ can be written

$$
{ }_{\bar{q}}^{(0)} \pi_{i}\left(\frac{\sqrt{t}}{r_{0}}\right) \sum_{r=1}^{\infty} r^{i} \lambda^{r} e^{-(q-\bar{q}) V_{\bar{t}} / r_{0}} / r_{0} !
$$

where $\lambda=q \sqrt{t} e^{-\bar{y} V \bar{t} / r_{0} / r_{0}}$. The maximal values of the $r^{\text {th }}$ terms in this sum for different values of the constants $q, \bar{q}, r_{0}$ have been calculated for $i=1.5 \sqrt{t}$ and for $\sqrt{t}=20$ and 30 . It was found that the $I I^{\text {th }}$ term was of the order of $10^{-16}$ for $\sqrt{t}=20$ and of $10^{-30}$ for $\sqrt{t}=30$. It, is, thus, to be expected that the number of terms needed for the calculation of the sum appearing in (23a), at least 
when (24a) has been inserted, is at most of the order of 20 , say, for $t=100,500$ and $\mathrm{I} 000$. The calculation of $\frac{}{a} \bar{X} F_{s}\left(c x, \frac{m \sqrt{\bar{t}}}{r_{0}}\right)$ can be performed by the formulae (2Ia)-(2Ic). It shall be remarked, that the number of terms needed for this calculation for a fixed value of $m$ must, on an average, be much lower than the numbers used in the numerical example of section I5. In this calculation we shall, namely, multiply ${ }^{(0)} \tau_{\rho}$ appearing in (2Ia) - (2IC) by the factor $\frac{\bar{q} m}{r_{0} \sqrt{t}}$, which on an average is less than I for $t=\mathrm{IOO}$, less than $\mathrm{I} / 2$ for $t=500$ and less than $\mathrm{I} / 20$ for $t=\mathrm{I} 000$. If the number of terms needed for the calculation by (2ra)-(2Ib), on this reason, should, on an average for each value of $m$ be reduced to $25 \%$ of the numbers used in the example of section 15 , the number of multiplications in the calculation of (2Ic) would be reduced to about $2 \%$ for each value of $m$. This should lead to a computation time for the calculation of (23a), with the insertion of (24a) of about the time required for the calculation by (2Ia) - (2Ic) according to section 15. Should the same assumption hold also, if $(24 \mathrm{~b})$ is inserted in (23a), we should, thus, be able to perform the computation of (23a) for both cases in nearly the same time of computation.

If in $(23 \mathrm{~b}),(24 \mathrm{a})$ and $(24 \mathrm{~b})$ are inserted, the sum appearing in (23b) reduces to closed expressions, for (24a) in the form of an exponential function and for $(24 \mathrm{~b})$ in the form of a power of a binomial. In this case we have only to calculate ${ }_{a}^{\left(\frac{1}{X}\right.} F_{s}\left(c x, \sqrt{t} / \boldsymbol{r}_{\mathbf{1}}\right)$ by the formulae (2Ia) - (2Ic), which according to the discussion in the last paragraph, requires about $5 \%$ of the time required according to section $\mathbf{I 5}$.

The stop loss risk premium can in the 4 cases considered in this section be computed with negligible additional work from the functions $\frac{\left(\frac{(x)}{a} \bar{G}\right.}{\bar{X}}$, the calculation of which has been included in the estimations given above, by using similar expressions to that given in (22).

19. The calculation of the distribution functions defined by (7), (8), (9) and the corresponding stop loss risk premiums based on processes, where all probability distributions of the number of changes involved are defined either by Poisson or by Polya distri- 
butions and where the change distribution for (7) and (9) is defined by ${ }_{b} V_{s}(x)$ and where the transform of the change distribution for (8) is defined as a weighted average of this function, can, thus, be performed on an electronic computer. For (9) it has been proved that for a given vector of the parameters in ${ }_{b} V_{s}(x)$ the calculations can be carried out in c: a $5 \mathrm{I} / 2$ hours for $s>2$, if the calculations for $s=\mathrm{I}$ will require 25 minutes. The same assertion is likely to hold for (7) for the compound Poisson process of type Poisson and in the corresponding case for (8). For the determination to what extent this is true, it seems easy to devise suitable experiments on the computer. For the compound process of type Polya and for the corresponding case of (8) the computation time required is materially less than that required in the other cases.

(based on a lecture read to the ASTIN Colloquium I962, Juan-lesPins)

\section{LITERATURE CITED}

Almer, B., 1957, Risk Analysis in Theory and Practical Statistics, Trans. $X V^{\text {th }}$ Int. Congr. Act., New York 1957, II, pp. 314-370.

_- 1962, Individual Risk Theory and Risk Statistics as applied to Fire Insurance, Report to the ASTIN Colloquium I962, Juan-les-Pins.

Ammeter, H., I948, A Generalization of the Collective Theory of Risk in Regard to Fluctuating Basic-Probabilities, Skand. Akt. Tidskr. 1948: 3-4, pp. I7I-I98.

(For further references see Philipson, 196I (b).)

CampaGne, C., 1962, Sur les événements en chaîne et la distribution binomiale négative généralisée, Rapport à l'ASTIN Colloquium I962, Juan-les-Pins.

Cramér, H., I955, Collective Risk Theory, A Survey from the Point of View of the Theory of Stochastic Processes, Stockholm, Skandia Jubilee Volume, 1955, 92 pages.

(For further references see Philipson, I96I (b).)

Delaporte, P., I959, Quelques problèmes de statistiques mathématique posés par l'assurance automobile et le bonus pour non sinistre, Bull. trim. de l'Inst. Act. Français, 65, pp. 87-Io2.

- 1960 , Un problème de tarification de l'assurance accidents d'automobiles examiné par la statistique mathématique, Trans. XVI Int. Congr. Act., Brussels I960, pp. I2I-I35.

Esscher, Fr., I932, On the Probability Function in the Collective Theory of Risk, Skand. Akt. Tidskr. I932, p. I75.

- I I96I-62, Memoranda on the Numerical Calculation of the Distribution Function in the Theory of Risk, Stockholm (unpublished).

Fisher, Sir R. and Yates, F., I938, Statistical Tables for Biological, Agricultural and Medical Research, London, Edinburgh.

Hofmann, M., I955, Über zusammengesetzte Poisson-Prozesse und ihre 
Anwendungen in der Unfallsversicherung, Mitteil. Vevein. Schweiz. Vers. Math., Vol. 55-3.

LundBerg, FILIP, I934, On the Numerical Application of the Collective Risk Theory, Stockholm, De Förenade Jubilee Volume, I934.

(For further references see Philipson, I96I (b).)

Lundberg, Ove, 1940, On Random Processes and Their Application to Sickness and Accident Statistics, Inaug. Diss. Uppsala, I 72 pages.

MatÉrn, B., I960, Spatial Variation, Stochastic Models and Their Applications to some Problems in Forest Surveys and other Sampling Investigations, Inaug. diss, Bull. Swed. Sta. Inst. Forestry Research, 49, I44 pages.

Pearson, E. S. and Hartley, H. O., 1954, Biometrika Tables for Statisticians, Vol. I., Cambridge Univ. Press.

Philipson, C., I960 (a) Note on the Application of Compound Poisson Processes to Sickness and Accident Statistics, The ASTIN Bull., Vol. I Part IV, pp. 225-237.

- I I 6 I (b) Note on the Background to the Subject: Theory of Risk, Fundamental Mathematics and Applications, The ASTIN Bull. Vol. I Part V, pp. 256-264. (based on Report I to the Rättvik Colloquium of ASTIN).

- I I I I (c) An Extension of the Models usually applied to the Theory of Risk, Report II to the Rättvik Colloquium of ASTIN Skand, Akt. Tidskr. 1961; 3-4 p. 223-239.

-_., I 96 I (d) A Generalization of the Stochastic Processes commonly applied to the Theory of Causalty Risk, Bull. de l'Inst. Int. de Statistique, $33^{\mathrm{e}}$ Session, Paris.

Segerdahl, C. O., 1955, When does Ruin occur in the Collective Theory of Risk, Skand. Akt. Tidskr. 1955: 1-2, pp. 22-36. (For further references see the paper quoted).

Slater, L. J., 1960, Confluent Hypergeometric Functions, Cambr. Univ. Press, 243 pages.

Thyrion, P., I960, Note sur les distribution "par grappes", Bull. Ass. Roy Act. Belg., I960: 6o, pp. 49-66. 